BMJ

Open

Gastroenterology

\section{Association between hepatic fat and subclinical vascular disease burden in the general population}

To cite: Cai X, Rospleszcz S, Mensel B, et al. Association between hepatic fat and subclinical vascular disease burden in the general population. BMJ Open Gastro 2021;8:e000709. doi:10.1136/ bmjgast-2021-000709

\section{- Additional supplemental material is published online only. To view, please visit the journal online (http://dx.doi. org/10.1136/bmjgast-2021- 000709).}

Received 14 May 2021 Accepted 27 July 2021
Check for updates

(C) Author(s) (or their employer(s)) 2021. Re-use permitted under CC BY-NC. No commercial re-use. See rights and permissions. Published by BMJ.

For numbered affiliations see end of article.

\section{Correspondence to}

Dr Jana Nano;

jana.nano@helmholtz-

muenchen.de

\section{ABSTRACT}

Objective It is still controversial if increased hepatic fat independently contributes to cardiovascular risk. We aimed to assess the association between hepatic fat quantified by MRI and various subclinical vascular disease parameters.

Design We included two cross-sectional investigations embedded in two independent population-based studies (Study of Health in Pomerania (SHIP): $n=1341$; Cooperative Health Research in the Region of Augsburg (KORA): $\mathrm{n}=386$ ). The participants underwent a whole-body MRI examination. Hepatic fat content was quantified by proton-density fat fraction (PDFF). Aortic diameters in both studies and carotid plaque-related parameters in KORA were measured with MRI. In SHIP, carotid intima-media thickness (CIMT) and plaque were assessed by ultrasound. We used (ordered) logistic or linear regression to assess associations between hepatic fat and subclinical vascular disease.

Results The prevalence of fatty liver disease (FLD) (PDFF $>5.6 \%$ ) was $35 \%$ in SHIP and $43 \%$ in KORA. In SHIP, hepatic fat was positively associated with ascending $(\beta$, $95 \% \mathrm{Cl} 0.06$ (0.04 to 0.08$)$ ), descending (0.05 (0.04 to $0.07)$ ) and infrarenal (0.02 (0.01 to 0.03$)$ ) aortic diameters, as well as with higher odds of plaque presence (OR, $95 \% \mathrm{Cl} 1.22$ (1.05 to 1.42)) and greater cIMT ( $\beta, 95 \%$ $\mathrm{Cl} 0.01$ (0.004 to 0.02)) in the age-adjusted and sexadjusted model. However, further adjustment for additional cardiometabolic risk factors, particularly body mass index, attenuated these associations. In KORA, no significant associations were found.

Conclusions The relation between hepatic fat and subclinical vascular disease was not independent of overall adiposity. Given the close relation of FLD with cardiometabolic risk factors, people with FLD should still be prioritised for cardiovascular disease screening.

\section{INTRODUCTION}

Fatty liver disease (FLD), defined as an ectopic fat accumulation $(\geq 5 \%)$ in the hepatocytes, constitutes the leading cause of chronic liver disease worldwide. ${ }^{1}$ With a growing prevalence of $2 \%-44 \%$ in the general population,

\section{Summary box}

What is already known about this subject?

- Fatty liver disease and subclinical vascular disease share several common cardiometabolic risk factors, such as type 2 diabetes and obesity. There is not yet population-based study to investigate the association between hepatic fat content and the expansion of aortic diameters. Epidemiological studies on the relation between fatty liver disease and atherosclerosis yielded controversial results.

What are the new findings?

- With data from the general population, we found that hepatic fat content measured with MRI was neither independently associated with greater aortic diameters nor the risk of carotid plaque, after adjusting for cardiometabolic risk factors, especially obesity.

How might it impact on clinical practice in the foreseeable future?

- Given that fatty liver disease is closely related to concurrent obesity, type 2 diabetes and other cardiometabolic disorders, people with fatty liver disease should still be recommended to monitor their cardiovascular risk factors and prioritised for cardiovascular disease screening.

clinical manifestations of FLD ranging from simple steatosis, steatohepatitis, fibrosis and eventually cirrhosis and hepatocellular carcinoma pose a substantial burden on healthcare systems. ${ }^{2}$ In particular, cardiovascular disease (CVD), one of the extrahepatic repercussions of FLD, remains to be the largest contributor of mortality among people with FLD. ${ }^{3}$

Prior to the clinical manifestation of CVD, subclinical vascular disease, representing pathological changes of various blood vessels, can provide important aetiological insights into early detection of CVD development. ${ }^{4}$ We have previously shown with our data 
that hepatic fat was positively associated with subclinical vascular parameters such as left ventricular remodelling index. ${ }^{5}$ Moreover, data from other population-based studies have also indicated a positive association between FLD and subclinical calcified plaque in different vessels. ${ }^{67}$

One understudied outcome of interest in the realm of subclinical vascular changes is aortic aneurysm, which represents a disproportionate dilation of aortic diameter (thoracic aorta diameter $\geq 5 \mathrm{~cm}$ or abdominal aorta diameter $\geq 3 \mathrm{~cm}$ ) that could result in life-threatening events such as aortic rupture or dissection. ${ }^{8}$ Several risk factors for the development of aortic aneurysm including age, increased body mass index (BMI) and hyperlipidaemia are mutually shared with FLD. ${ }^{9}$ However, whether hepatic fat is associated with expanding aortic dimeters, measured along multiple locations of the aorta, has not been previously investigated in a population-based setting.

Furthermore, the evidence is still inconsistent regarding whether hepatic fat represents an independent modifiable risk factor for subclinical atherosclerosis, such as carotid plaque. A meta-analysis reported that compared with people without FLD those who with FLD were almost $80 \%$ more likely to have carotid plaque detected by ultrasound. ${ }^{10}$ However, different criteria used for defining carotid plaque made their conclusions unconvincing. Ultrasound is less precise than CT or MRI in quantifying plaque calcification, which is particularly prone to progress to CVD events. ${ }^{11}$ Interestingly, two well-powered population-based studies could not show a link between FLD and carotid calcification measured by CT. ${ }^{12} 13$ On the other hand, most previous studies used either ultrasound or CT to define FLD, which are less sensitive than MRI when fat content is low. ${ }^{14}$

Therefore, we aimed to determine the association of hepatic fat quantified by dedicated whole-body MRI protocols with aortic diameters and carotid atherosclerosis measured by MRI or ultrasound in two wellcharacterised independent German studies.

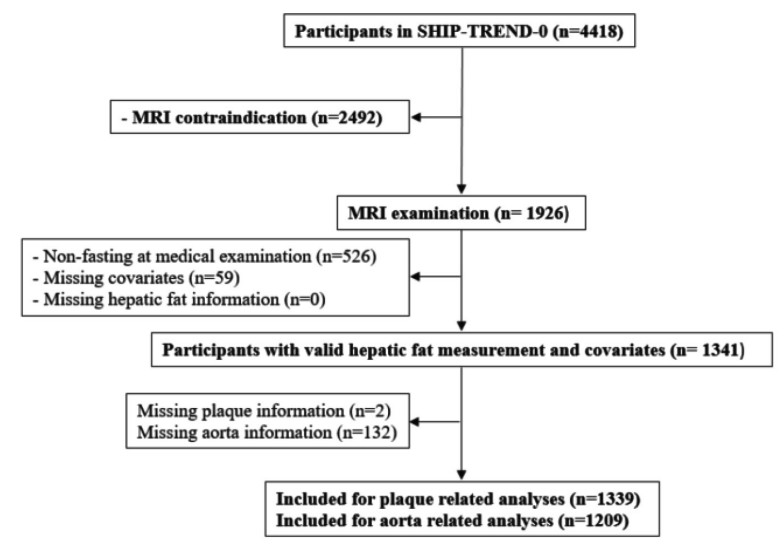

\section{METHODS}

\section{Study population}

The Study of Health in Pomerania

The baseline examination of the Study of Health in Pomerania (SHIP) - TREND-0 (SHIP-TREND-0 abbreviated as SHIP) was conducted between 2008 and 2012 and included participants from West Pomerania, northeastern part of Germany. The study design has previously been described in detail. ${ }^{15}$ Out of 8826 adults (20-79 years) randomly drawn from local population registries, a sample of 4420 eligible participants completed the baseline examination including personal interviews, laboratory measurements, ultrasonography and simple medical examinations. ${ }^{15}$ After excluding participants with MRI contraindication or who refused to participate $(n=2492)$, 1926 participants underwent a whole-body MRI examination. Exclusion criteria for MRI examination were described elsewhere. ${ }^{16}$ Further exclusion criteria for the present analysis included not fasting at the time of medical examination, missing values for hepatic fat measurements or any covariate $(n=585)$ leading to a total of 1341 participants for the final analyses (figure 1).

The Cooperative Health Research in the Region of Augsburg Study Cooperative Health Research in the Region of Augsburg (KORA) FF4 study, conducted between 2013 and 2014, was the second follow-up study of the KORA S4 survey (conducted between 1999 and 2001). We used the data from a subpopulation of the KORA FF4 study, which was originally selected for a nested case-control study to detect subclinical CVD in individuals with pre-diabetes and diabetes compared with those with normal glucose tolerance. Of all 4261 participants of the S4 survey, 2279 participants also participated in the FF4 study. The inclusion and exclusion criteria of the KORA-FF4-MRI (abbreviated as KORA) protocol were described elsewhere. ${ }^{17} \mathrm{~A}$ total of 400 participants underwent the whole-body MRI examination. They were free of overt CVD events, such as stroke, myocardial infarction and peripheral artery diseases. Finally, due to missing values in MRI-measured

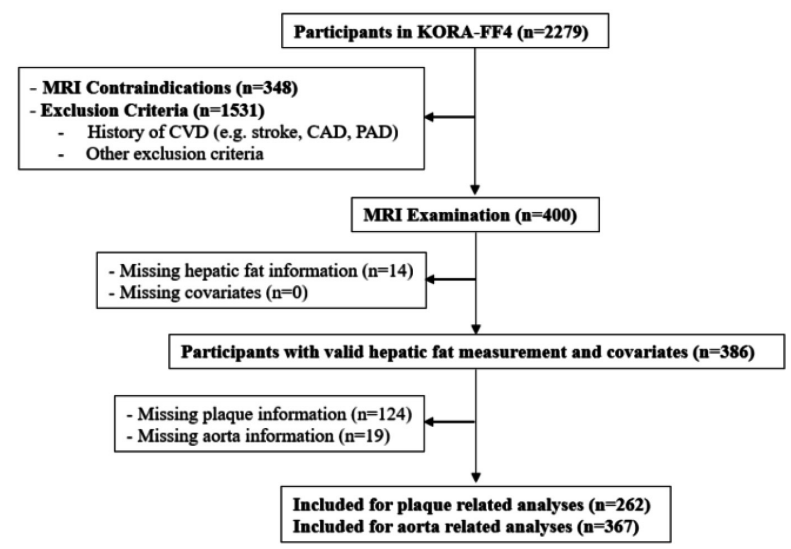

Figure 1 Flowchart of the study design. CAD, coronary artery disease; CVD, cardiovascular; KORA, Cooperative Health Research in the Region of Augsburg; PAD, peripherial artery disease; SHIP, Study of Health in Pomerania. 
hepatic fat content $(\mathrm{n}=14)$, the final population included in the analyses comprised 386 participants (figure 1).

Both studies comply with the Declaration of Helsinki. The SHIP study was approved by the Ethics Committee of University of Greifswald (BB 39/08). The KORA study was approved by the institutional review board of the Medical Faculty of Ludwig-Maximilian University Munich (06068). All participants provided written informed consent.

\section{Assessment of hepatic fat content and subclinical vascular parameters}

Hepatic fat content was estimated by MRI proton-density fat fraction (PDFF, \%) at the level of portal vein in both SHIP and KORA (see online supplemental materials 1 for the MRI device and sequence used). FLD was defined according to the Clinical Practice Guidelines for the management of non-alcoholic $\mathrm{FLD}^{18}$ : No FLD (PDFF $\leq 5.6 \%$ ) versus with FLD (PDFF $>5.6 \%$ ).

The diameters $(\mathrm{cm})$ of the ascending, descending and infrarenal aorta were measured in axial plane both in SHIP and KORA with MRI. As for carotid injury, in SHIP, the carotid intima-media thickness (cIMT) (mm) was measured with ultrasound and averaged over the left carotid artery (LCA) and the right carotid artery (RCA). Carotid plaque presence was adjudicated if any of the following three criteria was met: any focal thickening of the intima-media complex protruding into the vessel lumen, a focal increase in echogenicity with a homogeneously hyperechoic echotexture within an otherwise hypoechoic intima-media complex, a uniformly increased cIMT $(>1.3 \mathrm{~mm})$ without focal thickening. In KORA, presence and morphological composition of carotid plaque were determined in the MRI protocol. Mean wall thickness $(\mathrm{mm})$, lumen area $\left(\mathrm{mm}^{2}\right)$ and wall area $\left(\mathrm{mm}^{2}\right)$ were separately calculated for the LCA and the RCA. Normalised wall index (NWI), as calculated with wall area/(lumen area +wall area), describes the percentage of the wall surface in proportion to the total blood vessel surface. According to the presence of calcification and haemorrhage as well as wall thickness and wall eccentricity, plaque was differentiated and classified to type I, type III, type IV/V and type VI/VII with the criteria of the American Heart Association. ${ }^{19}$ Participants with type III, type IV/V or type VI/VII plaque were considered as having carotid plaque.

\section{Traditional cardiovascular risk factors and other covariates}

Other covariates were assessed in both studies, including traditional cardiovascular risk factors-such as BMI (kg/ $\mathrm{m}^{2}$ ), waist circumference $(\mathrm{cm})$, smoking status (smoker, ex-smoker, never smoker), physically active (yes, no), alcohol consumption (no intake, moderate intake, excessive intake: $\geq 20 \mathrm{~g} /$ day for women or $\geq 30 \mathrm{~g} /$ day for men), hypertension (yes, no), systolic blood pressure (SBP) $(\mathrm{mm} \mathrm{Hg})$, diastolic blood pressure $(\mathrm{mm} \mathrm{Hg})$, total cholesterol $(\mathrm{mmol} / \mathrm{L})$, high-density lipoprotein cholesterol (HDL-C) (mmol/L), low-density lipoprotein cholesterol (LDL-C) $(\mathrm{mmol} / \mathrm{L})$, triglycerides $(\mathrm{mmol} / \mathrm{L})$ and liver enzymes (aspartate aminotransferase (AST) $(\mu \mathrm{kat} / \mathrm{L})$, alanine aminotransferase (ALT) ( $\mu \mathrm{kat} / \mathrm{L})$, gamma-glutamyl transferase (GGT) $(\mu \mathrm{kat} / \mathrm{L}))$. Visceral adipose tissue (VAT) in litre was also measured in the whole-body MRI protocols. Participants were divided into three groups according to their glucose tolerance status: normoglycaemic (fasting glucose $<6.1 \mathrm{mmol} / \mathrm{L}$ and 2 hour glucose $<7.8 \mathrm{mmol} / \mathrm{L}$ ), pre-diabetes ( 2 hour glucose between $7.8 \mathrm{mmol} / \mathrm{L}$ and $11.0 \mathrm{mmol} / \mathrm{L}$ and normal fasting glucose or fasting glucose between 6.1 $\mathrm{mmol} / \mathrm{L}$ and $6.9 \mathrm{mmol} / \mathrm{L}$ and normal 2 hour glucose) and diabetes (fasting glucose $>6.9 \mathrm{mmol} / \mathrm{L}$ and/or 2 hour glucose $>11.0 \mathrm{mmol} / \mathrm{L}$ ) following each study protocols. ${ }^{15} 17$ Medication use within the last 7 days prior to the interview, such as use of antihypertensive ${ }^{20}$ and lipidlowering medication, was ascertained in both studies. In SHIP, history of CVD included events of myocardial infarction, stroke and angina pectoris. A detailed description of the MRI device measurements, the sequence used for hepatic fat content and subclinical vascular disease parameters and the definitions of other covariates are provided in online supplemental materials $1 .{ }^{15-27}$

\section{Statistical analyses}

We calculated the descriptive variables separately for SHIP and KORA as well as for the groups: participants with FLD (PDFF >5.6\%) versus participants without FLD (PDFF $\leq 5.6 \%$ ) within each study. For continuous variables, we displayed mean (SD) if they were normally distributed, and median (IQR) if the distribution was not normal. We show categorical variables with counts (percentages, \%). We used two-sample t-test for comparison of continuous variables and $\chi^{2}$ test for comparison of categorical variables. We log-transformed the variables that did not follow a normal distribution including triglycerides, ALT, AST, GGT in both studies, wall thickness of RCA and LCA, lumen area of RCA and LCA and wall area of RCA and LCA in KORA.

The coefficient estimates represent the change in subclinical disease parameters corresponding to one SD increase in log-transformed hepatic fat content. In both studies, we conducted linear regressions to examine the associations between hepatic fat content and continuous outcomes and logistic regression for categorical outcomes. The following three models were constructed for both SHIP and KORA. In model 1, we adjusted for age and sex. In model 2, we additionally included BMI. In model 3, we further adjusted for smoking, physical activity, alcohol intake, SBP, HDL-C, LDL-C, triglycerides, glucose tolerance status, history of CVD (in SHIP), use of antihypertensive medication and use of lipid-lowering medication. Considering the potential collinearity among the cardiometabolic covariates in model 3, we also calculated variance inflation factor for each covariate, ${ }^{28}$ which refuted the existence of strong collinearity among the covariates. The same models were conducted with FLD (yes vs no), as an important clinical endpoint of excessive 
hepatic fat. The interactions between hepatic fat content (or FLD) and diabetes as well as obesity (BMI $\geq 30 \mathrm{~kg}$ / $\mathrm{m}^{2}$ ) were examined by entering a multiplicative term (PDFF $\times$ diabetes or PDFF $\times$ obesity) in the models. The interaction between hepatic fat and history of CVD $(\mathrm{PDFF} \times \mathrm{CVD})$ was only assessed in SHIP.

In addition, sensitivity analyses were conducted in both studies excluding participants with excessive alcohol intake, defined as a daily alcohol intake $\geq 30 \mathrm{~g}$ for men and $\geq 20 \mathrm{~g}$ for women. ${ }^{18}$ Kühn et al have suggested another cut-off of PDFF ( $>5.1 \%$ ) for FLD based on histopathologic calibrations. ${ }^{21}$ We also conducted sensitivity analyses with FLD (yes vs no) defined by this cut-off in both studies. In order to examine the influence of visceral adiposity, we substituted BMI with VAT or waist circumference as a covariate in the models. Further sensitivity analyses excluding participants with a history of CVD were conducted in SHIP.

The significance level was set to a nominal $p$ value $<0.05$. Data analysis was performed with R-Studio V.4.0.2.

\section{RESULTS}

Table 1 summarises the baseline characteristics for the participants from the SHIP and KORA studies. Participants with a valid PDFF measurement consisted of 612 $(45.6 \%)$ men and $729(54.4 \%)$ women in SHIP and $223(57.8 \%)$ men and $163(42.2 \%)$ women in KORA. Mean age was lower in SHIP $(50.4 \pm 13.7$ years $)$ than in KORA (56.2 \pm 9.1 years). Moreover, participants in SHIP had lower waist circumference, were more physically active, suffered less from pre-diabetes and diabetes, but had higher prevalence of hypertension, compared with participants from KORA. Median PDFF and prevalence of FLD were lower in SHIP $(469,35.0 \%)$ than in KORA $(166,43.0 \%)$. We present demographic, anthropometric, lifestyle, cardiometabolic profiles stratified by FLD in both studies in online supplemental table 1 .

Aortic diameters were greater among SHIP participants (ascending aorta: $3.32 \pm 0.46 \mathrm{~cm}$; descending aorta: $2.46 \pm 0.35 \mathrm{~cm}$; infrarenal aorta: $1.85 \pm 0.23 \mathrm{~cm}$ ) than among KORA participants (ascending aorta: 2.96 \pm 0.41 $\mathrm{cm}$; descending aorta: $2.09 \pm 0.31 \mathrm{~cm}$; infrarenal aorta: $1.50 \pm 0.21 \mathrm{~cm})$. The presence of carotid plaque was more frequent in SHIP $(n=467,34.9 \%)$ than in KORA $(n=54$, $20.6 \%$ ). Morphological features of the plaque are listed in table 2. They are not comparable between the two studies due to different methods. Subclinical vascular parameters according to FLD in both studies are presented in online supplemental table 2.

\section{Associations between hepatic fat content and aortic diameters}

In SHIP, one SD increase in hepatic fat content was significantly associated with greater ascending $(\beta, 95 \% \mathrm{CI}$ $0.06(0.04$ to 0.08$))$, descending $(0.05(0.04$ to 0.07$))$ and infrarenal (0.02 (0.01 to 0.03)) aortic diameters in model 1. Further adjustment for BMI (model 2) substantially

\begin{tabular}{|c|c|c|}
\hline \multirow[t]{3}{*}{$\begin{array}{l}\text { Table } 1 \\
\text { KORA }\end{array}$} & f study particip & its in SHIP and \\
\hline & \multirow{2}{*}{$\begin{array}{l}\text { SHIP } \\
\text { Total }(\mathrm{N}=1341)\end{array}$} & \multirow{2}{*}{$\begin{array}{l}\text { KORA } \\
\text { Total }(\mathrm{N}=386)\end{array}$} \\
\hline & & \\
\hline Age (years) & $50.4(13.7)$ & $56.2(9.1)$ \\
\hline Women & $729(54.4 \%)$ & $163(42.2 \%)$ \\
\hline $\mathrm{BMI}\left(\mathrm{kg} / \mathrm{m}^{2}\right)$ & $27.4(4.4)$ & $28.1(4.9)$ \\
\hline Waist circumference $(\mathrm{cm})$ & $88.9(12.9)$ & $98.5(14.3)$ \\
\hline Physically active & $960(71.6 \%)$ & $230(59.6 \%)$ \\
\hline \multicolumn{3}{|l|}{ Smoking } \\
\hline Smoker & $289(21.6 \%)$ & 77 (19.9\%) \\
\hline Ex-smoker & $486(36.2 \%)$ & $169(43.8 \%)$ \\
\hline Never smoker & $566(42.2 \%)$ & $140(36.3 \%)$ \\
\hline \multicolumn{3}{|l|}{ Alcohol consumption } \\
\hline No intake & $164(12.2 \%)$ & $92(23.8 \%)$ \\
\hline Moderate intake & $1091(81.4 \%)$ & $191(49.5 \%)$ \\
\hline Excessive intake & $86(6.4 \%)$ & $103(26.7 \%)$ \\
\hline $\begin{array}{l}\text { Systolic blood pressure ( } \mathrm{mm} \\
\mathrm{Hg} \text { ) }\end{array}$ & $125.1(16.8)$ & $120.6(16.8)$ \\
\hline $\begin{array}{l}\text { Diastolic blood pressure (mm } \\
\mathrm{Hg})\end{array}$ & $76.6(9.7)$ & $75.3(10.0)$ \\
\hline Hypertension & $539(40.2 \%)$ & $132(34.2 \%)$ \\
\hline Total cholesterol (mmol/L) & $5.5(1.1)$ & $5.6(0.9)$ \\
\hline $\mathrm{HDL}-\mathrm{C}(\mathrm{mmol} / \mathrm{L})$ & $1.5(0.4)$ & $1.6(0.5)$ \\
\hline LDL-C (mmol/L) & $3.4(0.9)$ & $3.6(0.9)$ \\
\hline Triglycerides (mmol/L) & $1.2(0.9,1.7)$ & $1.2(0.9,1.8)$ \\
\hline ALT ( $\mu \mathrm{kat} / \mathrm{L})$ & $0.4(0.3,0.5)$ & $0.5(0.3,0.6)$ \\
\hline AST ( $\mu$ kat/L) & $0.3(0.2,0.4)$ & $0.4(0.3,0.5)$ \\
\hline GGT ( $\mu$ kat/L) & $0.5(0.4,0.7)$ & $0.5(0.3,0.7)$ \\
\hline \multicolumn{3}{|l|}{ Glucose tolerance status } \\
\hline Normoglycaemic & $932(69.5 \%)$ & $239(61.9 \%)$ \\
\hline Pre-diabetes & $281(21.0 \%)$ & 95 (24.6\%) \\
\hline Diabetes & $128(9.5 \%)$ & $52(13.5 \%)$ \\
\hline PDFF (\%) & $3.85(2.32,7.74)$ & $4.62(2.63,11,89)$ \\
\hline FLD & $469(35.0 \%)$ & $166(43.0 \%)$ \\
\hline $\begin{array}{l}\text { Antihypertensive medication } \\
\text { use }\end{array}$ & $369(27.5 \%)$ & $98(25.4 \%)$ \\
\hline Lipid-lowering medication use & $109(8.1 \%)$ & $41(10.6 \%)$ \\
\hline History of CVD & $93(6.9 \%)$ & NA \\
\hline
\end{tabular}

Values are expressed as the mean (SD) for normally distributed continuous variables or median (IQR) for non-normally distributed continuous variables, or $\mathrm{n}(\%)$ for categorical variables.

Hepatic fat content was quantified on the level of portal vein by MRI PDFF. FLD (PDFF >5.6\%) was defined according to the European Association for the Study of the Liver (EASL)-European Association for the Study of Diabetes (EASD)-European Association for the Study of Obesity (EASO) Clinical

Practice Guidelines for the management of non-alcoholic fatty liver disease. ALT, alanine aminotransferase; AST, aspartate aminotransferase; BMI, body mass index; CVD, cardiovascular disease; FLD, fatty liver disease; GGT, gamma-glutamyl transferase; HDL-C, high-density lipoprotein cholesterol; KORA, Cooperative Health Research in the Region of Augsburg; LDL-C, lowdensity lipoprotein cholesterol; PDFF, proton density fat fraction; SHIP, Study of Health in Pomerania.

attenuated the estimates. Adding other cardiometabolic risk factors in model 3 changed the results only marginally. 
Table 2 Subclinical vascular disease parameters among participants in SHIP and KORA

\begin{tabular}{|c|c|c|}
\hline & SHIP & KORA \\
\hline & Total $(\mathrm{N}=1341)$ & Total $(\mathrm{N}=386)$ \\
\hline $\begin{array}{l}\text { Ascending aorta diameter } \\
(\mathrm{cm})\end{array}$ & $3.32(0.46)$ & $2.96(0.41)$ \\
\hline $\begin{array}{l}\text { Descending aorta diameter } \\
(\mathrm{cm})\end{array}$ & $2.46(0.35)$ & $2.09(0.31)$ \\
\hline Infrarenal aorta diameter $(\mathrm{cm})$ & $1.85(0.23)$ & $1.50(0.21)$ \\
\hline Plaque presence & 467 (34.9\%) & $54(20.6 \%)$ \\
\hline Type of plaque & NA & \\
\hline AHA type I & & 208 (79.4\%) \\
\hline AHA type III & & $38(14.5 \%)$ \\
\hline AHA type V & & $10(3.8 \%)$ \\
\hline AHA type VI or VII & & $6(2.3 \%)$ \\
\hline $\begin{array}{l}\text { Carotid intima-media } \\
\text { thickness }(\mathrm{mm})\end{array}$ & $0.60(0.14)$ & NA \\
\hline Wall thickness, LCA (mm) & NA & $0.73(0.69,0.79)$ \\
\hline Wall thickness, RCA (mm) & NA & $0.73(0.69,0.81)$ \\
\hline Lumen area, LCA $\left(\mathrm{mm}^{2}\right)$ & NA & $17.54(14.02,21.61)$ \\
\hline Lumen area, RCA $\left(\mathrm{mm}^{2}\right)$ & NA & $16.40(13.14,20.68)$ \\
\hline Wall area, LCA $\left(\mathrm{mm}^{2}\right)$ & NA & $12.39(10.72,14.03)$ \\
\hline Wall area, $\mathrm{RCA}\left(\mathrm{mm}^{2}\right)$ & NA & $12.18(10.36,14.06)$ \\
\hline NWI, LCA & NA & $0.44(0.05)$ \\
\hline NWI, RCA & NA & $0.45(0.05)$ \\
\hline
\end{tabular}

Values are expressed as the mean (SD) for continuous variables, or $\mathrm{n}(\%)$ for categorical variables.

Hepatic fat content was quantified on the level of portal vein by MRI PDFF. Plaque was detected using ultrasound in SHIP and MRI in KORA. Number of missing values for each outcome variable is shown in online supplemental table 2.

AHA, American Heart Association; KORA, Cooperative Health Research in the Region of Augsburg; LCA, left carotid artery; NA, not applicable; NWI, normalised wall index, calculated as wall area/(lumen area + wall area); PDFF, proton density fat fraction; RCA, right carotid artery; SHIP, Study of Health in Pomerania.

In KORA, hepatic fat content and aortic diameters were not significantly associated in all three models (table 3 ) (model 3: ascending aorta: $\beta, 95 \%$ CI $-0.04(-0.09$ to $0.02)$; descending aorta: -0.03 ( -0.07 to 0.01$)$ and infrarenal aorta: -0.01 ( -0.04 to 0.01$)$ ).

\section{Associations between hepatic fat content and carotid plaque and related parameters}

In SHIP, one SD increase of hepatic fat content was associated with higher odds of plaque presence (OR, 95\% CI $1.22(1.05$ to 1.42$)$ ) and greater CIMT ( $\beta, 95 \%$ CI 0.01 $(0.004$ to 0.02$))$ in model 1. Further adjustment for BMI in model 2 attenuated these associations (plaque presence: OR, $95 \%$ CI 1.13 (0.95 to 1.33) and cIMT: $\beta, 95 \%$ CI 0.002 (-0.01 to 0.01$))$. Further adjustment for other cardiometabolic risk factors (model 3) changed the results only marginally.

In KORA, we did not observe any significant associations between hepatic fat content and plaque-related outcomes including plaque presence (model 3: OR, 95\% CI 0.80 $(0.49$ to 1.29$))$ and plaque type $(0.73$ ( 0.45 to 1.19$))$ (table 3). Regression analyses with the morphological features of plaque revealed non-significant estimates in all models (table 3).

\section{Sensitivity analysis}

The interaction terms between hepatic fat content and diabetes/obesity/CVD in SHIP were not significant. In KORA, we found significant interactions between hepatic fat content and diabetes for plaque presence ( $p_{\text {PDFFxdia- }}$ $\left.{ }_{\text {betes }}=0.031\right)$ and plaque type $\left(p_{\text {pDFFxdiabetes }}=0.020\right)$. Due to the enrichment of participants with altered glucose metabolism (pre-diabetes and diabetes) in KORA, we did subgroup analyses stratified by glycaemic status (normoglycaemia vs pre-diabetes or diabetes). Among participants with altered glucose metabolism, carotid plaque presence (OR, 95\% CI 0.44 (0.21 to 0.91$)$ ) and plaque type $(0.39$ ( 0.19 to 0.79$))$ were inversely associated with hepatic fat content after full adjustment (online supplemental table 3).

Considering the role of alcohol intake in the pathogenesis of FLD, we also conducted sensitivity analyses excluding participants with excessive alcohol intake. ${ }^{18}$ The latter did not change the estimates except for NWI of the RCA in KORA, which was significantly inversely associated with hepatic fat only in model 3 ( $\beta, 95 \%$ CI -0.01 $(-0.02$ to -0.0004$))$. Very likely this represents a spurious findings due to the large number of tests (online supplemental table 4). Therefore, most likely we can generalise our results to the context of non-alcoholic FLD.

Regression analyses with FLD (yes vs no) as exposure, defined by either cut-off of PDFF ( $5.6 \%$ or $5.1 \%$ ), showed similar non-significant coefficient estimates in both studies (online supplemental table 5). Replacing BMI with either VAT or waist circumference as a covariate did not influence the results in both studies (online supplemental table 5). In SHIP, sensitivity analyses excluding participants with a history of CVD hardly affected the results (online supplemental table 6).

\section{DISCUSSION}

In this investigation comprising two cross-sectional investigations embedded in two independent population-based studies, we found no association between increasing hepatic fat content measured by MRI and parameters of subclinical vascular disease, including (ascending, descending, infrarenal) aortic diameters and carotid plaque presence and its morphological features. The link between hepatic fat content and subclinical vascular parameters was mainly driven by general adiposity and other cardiometabolic risk factors, such as hyperlipidemia and hypertension, which very often coexist with FLD. This indicates that the role of hepatic fat on subclinical vascular burden might be rather a reflection of worsened cardiometabolic profile.

\section{Hepatic fat content and aortic diameters}

The present investigation is the first to report the association between increasing hepatic fat and aortic diameters in a population-based setting. A clinical study from 


\begin{tabular}{|c|c|c|c|c|c|c|c|}
\hline & $\mathbf{N}$ & Model 1 & $P$ value & Model 2 & $P$ value & Model 3* & $P$ value \\
\hline \multicolumn{8}{|l|}{ SHIP } \\
\hline Ascending aorta $(\mathrm{cm})$ & 1209 & $0.06(0.04$ to 0.08$)$ & $<0.001$ & $0.01(-0.02$ to 0.03$)$ & 0.469 & $0.001(-0.02$ to 0.03$)$ & 0.913 \\
\hline Descending aorta $(\mathrm{cm})$ & 1209 & 0.05 (0.04 to 0.07$)$ & $<0.001$ & $0.01(-0.01$ to 0.02$)$ & 0.228 & $0.01(-0.01$ to 0.02$)$ & 0.533 \\
\hline \multirow[t]{2}{*}{ Plaque presence } & 1339 & $1.22(1.05$ to 1.42$)$ & 0.008 & 1.13 (0.95 to 1.33$)$ & 0.178 & $1.03(0.85$ to 1.25$)$ & 0.753 \\
\hline & & $\beta, 95 \% \mathrm{Cl}$ & & $\beta, 95 \% \mathrm{Cl}$ & & $\beta, 95 \% \mathrm{Cl}$ & \\
\hline $\begin{array}{l}\text { Carotid intima-media- } \\
\text { thickness }(\mathrm{mm})\end{array}$ & 1339 & 0.01 (0.004 to 0.02$)$ & 0.002 & $0.002(-0.01$ to 0.01$)$ & 0.538 & $-0.003(-0.01$ to 0.004$)$ & 0.401 \\
\hline \multicolumn{8}{|l|}{ KORA } \\
\hline Infrarenal aorta $(\mathrm{cm})$ & 367 & $0.02(-0.004$ to 0.03$)$ & 0.115 & $-0.01(-0.03$ to 0.02$)$ & 0.536 & $-0.01(-0.04$ to 0.01$)$ & 0.349 \\
\hline Carotid plaque & & OR, $95 \% \mathrm{Cl}$ & & OR, $95 \% \mathrm{CI}$ & & OR, $95 \% \mathrm{Cl}$ & \\
\hline Plaque presence & 262 & 0.93 (0.66 to 1.32$)$ & 0.676 & 0.84 (0.56 to 1.25$)$ & 0.390 & 0.80 (0.49 to 1.29$)$ & 0.354 \\
\hline \multirow[t]{2}{*}{ Plaque type } & 262 & 0.93 (0.66 to 1.31$)$ & 0.692 & $0.80(0.53$ to 1.20$)$ & 0.274 & 0.73 (0.45 to 1.19$)$ & 0.206 \\
\hline & & $\beta, 95 \% \mathrm{Cl}$ & & $\beta, 95 \% \mathrm{Cl}$ & & $\beta, 95 \% \mathrm{Cl}$ & \\
\hline Wall thickness, LCA (mm) & 251 & 0.01 (-0.01 to 0.03$)$ & 0.474 & $-0.01(-0.03$ to 0.01$)$ & 0.186 & $-0.02(-0.04$ to 0.01$)$ & 0.179 \\
\hline Wall thickness, RCA (mm) & 257 & 0.01 (-0.01 to 0.02$)$ & 0.534 & $-0.01(-0.03$ to 0.01$)$ & 0.193 & $-0.01(-0.03$ to 0.01$)$ & 0.287 \\
\hline Lumen area, LCA $\left(\mathrm{mm}^{2}\right)$ & 255 & $0.02(-0.03$ to 0.06$)$ & 0.481 & $-0.01(-0.06$ to 0.05$)$ & 0.841 & $0.001(-0.06$ to 0.06$)$ & 0.968 \\
\hline Lumen area, RCA $\left(\mathrm{mm}^{2}\right)$ & 262 & $0.03(-0.02$ to 0.08$)$ & 0.266 & $-0.003(-0.06$ to 0.05$)$ & 0.915 & $0.04(-0.03$ to 0.10$)$ & 0.271 \\
\hline Wall area, LCA $\left(\mathrm{mm}^{2}\right)$ & 251 & $0.01(-0.02$ to 0.05$)$ & 0.414 & $-0.02(-0.06$ to 0.02$)$ & 0.288 & $-0.02(-0.06$ to 0.03$)$ & 0.448 \\
\hline
\end{tabular}

Model 1: adjusted for age, sex

Model 2: model 1+BMl

Model 3: model 2+smoking status, physical activity, alcohol intake, systolic blood pressure, HDL-C, LDL-C, triglycerides, glucose tolerance status, use of antihypertensive

medication, use of lipid-lowering medication.

Results with $p$ value $<0.05$ are shown in bold. The coefficient estimates represent the change in subclinical disease parameters with a SD increment of log-transformed hepatic fat content.

*Model 3 in SHIP was additionally adjusted for history of cardiovascular diseases.

$\beta$, $\beta$-estimates from linear regression; BMI, body mass index; HDL-C, high-density lipoprotein cholesterol; KORA, Cooperative Health Research in the Region of Augsburg; LCA, left carotid artery; LDL-C, low-density lipoprotein cholesterol; NWI, normalised wall index, calculated as wall area/(lumen area+wall area); RCA, right carotid artery; SHIP, Study of Health in Pomerania.

Mahamid et al, including data from 495 hospitalised patients with abdominal aortic aneurysm (AAA) diagnosed with either ultrasound or CT and 500 matched controls, reported a positive relation between FLD and AAA occurrence adjusted for age, smoking, BMI and metabolic syndrome. ${ }^{9}$ However, these results should be interpreted with caution given the highly selective sample of hospitalised patients, who were diagnosed with a clinically relevant and more advanced stage of infrarenal aorta dilation $(\geq 3 \mathrm{~cm}),{ }^{29}$ which could have biased the results.

FLD and AAA share common pathophysiological risk factors such as adiposity, insulin resistance and inflammation. In our study, the adjustment for BMI substantially attenuated the associations of hepatic fat with aortic diameters. Accordingly, previous animal studies have shown that leptin secreted by adipocytes could promote proinflammatory cytokines, for example, interleukin-18, binding to their receptors on aortic smooth muscle cells. Consequently, adipocytes could enhance aortic inflammation and exacerbate AAA development. ${ }^{30}$ Because of the close relation between hepatic fat and central obesity, we also substituted BMI with better measurements of visceral adiposity such as waist circumference and VAT, the latter measured with MRI. The results did not change. This indicates that higher general or central adiposity, which often coexists with FLD, confounds the association between hepatic fat content and increasing aortic diameters. Therefore, an independent effect of hepatic fat in the aetiology of AAA could not be shown.

\section{Hepatic fat content, carotid plaque and related parameters}

Previous studies have yielded controversial results regarding the association between FLD and subclinical carotid atherosclerosis. The largest meta-analysis to date with studies assessing FLD and carotid plaque defined by 
ultrasound revealed a positive association between these two parameters. ${ }^{10}$ Although cIMT provides insight into continuous change of vessel walls over time, the clinical cut-off point of cIMT that distinguishes people with increased cardiovascular risk varied among the studies. Moreover, the meta-analysis included different methods for determining FLD, either by ultrasound or biopsy. Although biopsy remains the gold standard for diagnosing FLD, it is prone to sampling error. ${ }^{31}$ Ultrasound has limited performance in detecting FLD at a milder stage. ${ }^{31}$ Due to these heterogeneities in measuring methods, the conclusion of this meta-analysis is less convincing and remains a controversial.

On the other side, results from two population-based studies suggested that FLD is not an independent risk factor for subclinical carotid plaque. ${ }^{12}{ }^{13}$ In an investigation using data from the Rotterdam Study, Wolff et al found that higher hepatic fat measured by CT was related to increasing volumes of coronary artery, yet not to carotid artery calcification. ${ }^{12}$ Similar results were found in another study using CT scans from Koo et al, where they showed that FLD was positively associated with calcification in thoracic aorta and coeliac trunk, but not carotid artery. The distinct atherogenic effects of FLD on different vascular beds may reflect different underlying mechanisms. The location of subclinical alterations with regards to FLD merits emphasis in future studies.

In line with previous literature in population-based setting, ${ }^{12} 13$ we found no evidence of an independent role of hepatic fat on carotid plaque development. An observation from Di Costanzo et al found that cIMT only increased among people with FLD sharing other metabolic abnormalities, but not in people with FLD without metabolic abnormalities, compared with controls without FLD. ${ }^{32}$ It might be plausible that FLD does not add to the burden of carotid atherosclerosis, unless it coexists with metabolic abnormalities. In addition, other studies in settings enriched with patients with type 2 diabetes found no independent association between FLD and subclinical carotid plaque, after adjustment for insulin resistance. ${ }^{33-35}$ It has been indicated by previous studies that insulin resistance could be a major factor increasing cardiovascular risks of people with FLD ${ }^{36}$ In the Tübingen Diabetes Family Study with participants at higher risk of diabetes, researchers have shown that insulin resistance increased with higher hepatic fat content measured by MRI ${ }^{37}$ mainly caused by imbalance of adipocytokines. ${ }^{38}$ Moreover, insulin resistance could promote inflammation and endothelial dysfunction, two major factors of an atherogenic environment. ${ }^{39}$ These evidences pointed out that ectopic fat accumulation in liver might be regarded as a hepatic manifestation of metabolic dysfunction.

While certainly not wanting to overlook the clinical relevance of FLD, focus should be laid on assessing and treating metabolic dysfunction of FLD people as an attempt to reduce CVD risk. In light of the new definition for metabolic dysfunction-associated FLD, the level of cardiovascular risks among metabolically unhealthy people with FLD is equally high, regardless of obesity. ${ }^{40} 41$ Although we did not find an independent role of hepatic fat on subclinical vascular disease that extends beyond overall adiposity and other cardiometabolic risk factors, we still think it is important for continue screening patients with FLD for CVD.

\section{Strengths and limitations}

This is the first study to investigate the association between hepatic fat content/FLD and subclinical vascular changes in both aorta and carotid artery by whole-body MRI examination. With data from two cross-sectional investigations embedded in population-based cohorts, we were able to show the consistency of the associations. Both studies collected data on a variety of cardiometabolic and lifestyle risk factors that were considered in the model adjustment and allowed for examining potential differences in subgroups in the sensitivity analyses. Moreover, MRI demonstrates the best overall performance in determining FLD, ${ }^{42}$ and it is also highly sensitive and specific for carotid plaque imaging. ${ }^{43}$

Some limitations need to be addressed. The MRI protocols used in SHIP and KORA were not able to distinguish more advanced stage of FLD involving fibrosis (using, eg, elastography) from simple steatosis, which could potentially modify the association between hepatic fat and subclinical vascular diseases. Despite the two different measuring modalities for carotid plaque (ultrasound in SHIP and MRT in KORA), the consistent null result indicated that the lack of association is unlikely to be due to methodological discrepancy. Additionally, due to the cross-sectional design of our study, we could only capture the relationship between hepatic fat content and subclinical vascular parameters at one point of time. This design does not allow for interpretation on the directionality of associations. Given that fat accumulation in liver is modifiable ${ }^{38}$ whether the elevation/amelioration of hepatic fat could accelerate/reverse the development of subclinical vascular diseases over time warrants further investigation.

\section{CONCLUSION}

We found that the associations between hepatic fat measured with MRI and subclinical vascular disease such as aortic diameters and subclinical atherosclerosis parameters were not independent of overall adiposity and a worsened cardiometabolic risk profile. Given the close relation of hepatic fat to other cardiometabolic risk factors, such as obesity, dyslipidaemia, hypertension and diabetes, we cannot afford to overlook the role of FLD on CVD development. Therefore, people with FLD should still be strongly advised to modify their CVD risks, such as overall adiposity, which can be targeted with lifestyle interventions. Well-powered prospective cohort studies with state-of-the-art imaging modalities are needed to better understand the contribution of hepatic fat in subclinical vascular disease development. 
Author affiliations

${ }^{1}$ Institute of Epidemiology, Helmholtz Center Munich German Research Center for Environmental Health, Neuherberg, Germany

${ }^{2}$ Institute for Medical Information Processing, Biometry, and Epidemiology-IBE, Pettenkofer School of Public Health, Ludwig-Maximilians-Universitat Munchen, Munich, Germany

${ }^{3}$ Department of Epidemiology, Institute for Medical Information Processing, Biometry and Epidemiology—-BBE, Ludwig-Maximilians-Universitat Munchen, Munich, Germany

${ }^{4}$ German Center for Cardiovascular Disease Research (DZHK), partner site Munich Heart Alliance, Munich, Germany

${ }^{5}$ Institute of Diagnostic and Interventional Radiology, Jena University Hospital, Jena, Germany

${ }^{6}$ Department of Neurology, University Medicine Greifswald, Greifswald, Germany ${ }^{7}$ Institute and Policlinic for Diagnostic and Interventional Radiology, University Hospital Carl-Gustav-Carus, Dresden University of Technology, Dresden, Germany ${ }^{8}$ Department of Internal Medicine A, University Medicine Greifswald, Greifswald, Germany

${ }^{9}$ Department of Neuroradiology, Faculty of Medicine, Medical Center-University of Freiburg, Freiburg, Germany

${ }^{10}$ Department of Radiology, University Hospital, LMU Munich, Munich, Bayern, Germany

${ }^{11}$ Department of Diagnostic and Interventional Radiology, Medical Center-University of Freiburg, Freiburg, Germany

${ }^{12}$ Institute for Biometrics and Epidemiology, German Diabetes Center Leibniz Institute for Diabetes Research at Heinrich Heine University Düsseldorf, Düsseldorf, Germany

${ }^{13}$ Partner site Düsseldorf, German Center for Diabetes Research, Neuherberg,

Germany

${ }^{14}$ Department of Endocrinology and Diabetology, Medical Faculty, Heinrich Heine University Düsseldorf, Düsseldorf, Germany

${ }^{15}$ Institute for Clinical Diabetology, German Diabetes Center, German Diabetes Center Leibniz Institute for Diabetes Research at Heinrich Heine University Düsseldorf, Düsseldorf, Germany

${ }^{16}$ Department of Medicine II, University Hospital, LMU Munich, Munich, Germany

${ }^{17}$ Institute of Diagnostic Radiology and Neuroradiology, University Medicine Greifswald, Greifswald, Germany

${ }^{18}$ Institute for Medical Information Processing, Biometry, and Epidemiology—IBE, Ludwig Maximilians University Munich, Munich, Germany

${ }^{19}$ Partner site Munich-Neuherberg, German Center for Diabetes Research, Neuherberg, Germany

${ }^{20}$ Institute for Community Medicine, SHIP/Clinical-Epidemiological Research, University Medicine Greifswald, Greifswald, Germany

${ }^{21}$ Partner site Greifswald, German Center for Cardiovascular Disease Research, Greifswald, Germany

Contributors All persons listed as coauthors have contributed to preparing the manuscript: $X C$ has designed the analyses, interpreted the data and drafted the manuscript; SR has contributed to the conception and design of the data analyses; $\mathrm{JN}$ has contributed to conception, design and interpretation of the data and approval of the manuscript; BM, US, J-PK, AAA, CS, RL, CLS, WR, MR, SH, RB, FB, $\mathrm{AP}, \mathrm{BT}$ and $\mathrm{HV}$ have contributed substantially to the interpretation of the data and have critically revised the manuscript for important intellectual content. BT and HV are responsible for the overall content as guarantors.

Funding The Study of Health in Pomerania (SHIP) is part of the Community Medicine Research Network of the University Medicine Greifswald, which is supported by the German Federal Ministry of Education and Research (BMBF, grant numbers: $01 Z Z 96030$ and 01ZZ0701). MRI scans in SHIP-TREND-0 have been supported by a joint grant from Siemens Healthineers, Erlangen, Germany and the Federal State of Mecklenburg-West Pomerania. The KORA research platform and the MONICA/KORA Augsburg studies are financed by the Helmholtz Zentrum München, German Research Center for Environmental Health $(\mathrm{GmbH})$, which is funded by the German Federal Ministry of Education and Research (BMBF) and by the State of Bavaria. The KORA-MRI sub-study received funding by the German Research Foundation (DFG, grant number: BA 4233/4-1), and German Centre for Cardiovascular Disease Research (DZHK, Berlin, Germany, grant numbers: $81 X 2600209$ and $81 X 2600214)$. This study was funded by a grant from the German Center for Diabetes Research (DZD-NEXT) to Jana Nano

Competing interests None declared.
Patient consent for publication Not required.

Provenance and peer review Not commissioned; externally peer reviewed.

Data availability statement Data are available upon reasonable request. The datasets generated and/or analysed during the current study are not publicly available due to data protection reasons. The data are available in an anonymised form on reasonable request to the corresponding author.

Supplemental material This content has been supplied by the author(s). It has not been vetted by BMJ Publishing Group Limited (BMJ) and may not have been peer-reviewed. Any opinions or recommendations discussed are solely those of the author(s) and are not endorsed by BMJ. BMJ disclaims all liability and responsibility arising from any reliance placed on the content. Where the content includes any translated material, BMJ does not warrant the accuracy and reliability of the translations (including but not limited to local regulations, clinical guidelines, terminology, drug names and drug dosages), and is not responsible for any error and/or omissions arising from translation and adaptation or otherwise.

Open access This is an open access article distributed in accordance with the Creative Commons Attribution Non Commercial (CC BY-NC 4.0) license, which permits others to distribute, remix, adapt, build upon this work non-commercially, and license their derivative works on different terms, provided the original work is properly cited, appropriate credit is given, any changes made indicated, and the use is non-commercial. See: http://creativecommons.org/licenses/by-nc/4.0/.

ORCID iD

Xinting Cai http://orcid.org/0000-0003-1837-3289

\section{REFERENCES}

1 Younossi Z, Anstee QM, Marietti M, et al. Global burden of NAFLD and NASH: trends, predictions, risk factors and prevention. Nat Rev Gastroenterol Hepatol 2018;15:11-20.

2 Blachier M, Leleu H, Peck-Radosavljevic M, et al. The burden of liver disease in Europe: a review of available epidemiological data. $J$ Hepatol 2013;58:593-608.

3 Adams LA, Anstee QM, Tilg $\mathrm{H}$, et al. Non-alcoholic fatty liver disease and its relationship with cardiovascular disease and other extrahepatic diseases. Gut 2017;66:1138-53.

4 Ingelsson E, Sullivan LM, Fox CS, et al. Burden and prognostic importance of subclinical cardiovascular disease in overweight and obese individuals. Circulation 2007;116:375-84.

5 Schlett CL, Lorbeer R, Arndt C, et al. Association between abdominal adiposity and subclinical measures of left-ventricular remodeling in diabetics, prediabetics and normal controls without history of cardiovascular disease as measured by magnetic resonance imaging: results from the KORA-FF4 study. Cardiovasc Diabetol 2018;17:88.

6 Mellinger JL, Pencina KM, Massaro JM, et al. Hepatic steatosis and cardiovascular disease outcomes: an analysis of the framingham heart study. J Hepatol 2015;63:470-6.

7 Kapuria D, Takyar VK, Etzion O, et al. Association of hepatic steatosis with subclinical atherosclerosis: systematic review and meta-analysis. Hepatol Commun 2018;2:877-87.

8 Kälsch H, Lehmann N, Möhlenkamp S, et al. Body-surface adjusted aortic reference diameters for improved identification of patients with thoracic aortic aneurysms: results from the population-based heinz Nixdorf recall study. Int J Cardiol 2013;163:72-8.

9 Mahamid M, Khoury T, Mahamid B, et al. The interplay between abdominal aortic aneurysm, metabolic syndrome and fatty liver disease: a retrospective case-control study. Diabetes Metab Syndr Obes 2019;12:12.

10 Madan SA, John F, Pyrsopoulos N, et al. Nonalcoholic fatty liver disease and carotid artery atherosclerosis in children and adults: a meta-analysis. Eur J Gastroenterol Hepatol 2015;27:1237-48.

11 Syed MB, Fletcher AJ, Forsythe RO, et al. Emerging techniques in atherosclerosis imaging. Br J Radiol 2019;92:20180309.

12 Wolff $L$, Bos D, Murad SD, et al. Liver fat is related to cardiovascular risk factors and subclinical vascular disease: the rotterdam study. Eur Heart J Cardiovasc Imaging 2016;17:1361-7.

13 Koo BK, Allison MA, Criqui MH, et al. The association between liver fat and systemic calcified atherosclerosis. J Vasc Surg 2020;71:e4.:204-11.

14 Li Q, Dhyani M, Grajo JR, et al. Current status of imaging in nonalcoholic fatty liver disease. World J Hepatol 2018;10:530-42.

15 Völzke H, Alte D, Schmidt CO, et al. Cohort profile: the study of health in pomerania. Int J Epidemiol 2011;40:294-307. 
16 Kühn J-P, Meffert P, Heske C, et al. Prevalence of fatty liver disease and hepatic iron overload in a northeastern German population by using quantitative MR imaging. Radiology 2017;284:706-16.

17 Bamberg F, Hetterich H, Rospleszcz S, et al. Subclinical disease burden as assessed by whole-body MRI in subjects with prediabetes, subjects with diabetes, and normal control subjects from the general population: the KORA-MRI study. Diabetes 2017;66:158-69.

18 European Association for the Study of the Liver (EASL), European Association for the Study of Diabetes (EASD), European Association for the Study of Obesity (EASO). EASL-EASD-EASO clinical practice guidelines for the management of non-alcoholic fatty liver disease. $J$ Hepatol 2016;64:1388-402.

19 Cai J-M, Hatsukami TS, Ferguson MS, et al. Classification of human carotid atherosclerotic lesions with in vivo multicontrast magnetic resonance imaging. Circulation 2002;106:1368-73.

20 Scholze J. Empfehlungen zur hochdruckbehandlung in der praxis. Notfall \& Hausarztmedizin 2005;31:152-9.

21 Kühn J-P, Hernando D, Muñoz del Rio A, et al. Effect of multipeak spectral modeling of fat for liver iron and fat quantification: correlation of biopsy with MR imaging results. Radiology 2012;265:133-42.

22 Zhong X, Nickel MD, Kannengiesser SAR, et al. Liver fat quantification using a multi-step adaptive fitting approach with multiecho GRE imaging. Magn Reson Med 2014;72:1353-65.

23 Lorbeer R, Bayerl C, Auweter S, et al. Association between MRIderived hepatic fat fraction and blood pressure in participants without history of cardiovascular disease. J Hypertens 2017;35:737-44

24 Mensel B, Heßelbarth L, Wenzel M, et al. Thoracic and abdominal aortic diameters in a general population: MRI-based reference values and association with age and cardiovascular risk factors. Eur Radiol 2016;26:969-78.

25 Saam T, Ferguson MS, Yarnykh VL, et al. Quantitative evaluation of carotid plaque composition by in vivo MRI. Arterioscler Thromb Vasc Biol 2005;25:234-9.

26 Holle R, Happich M, Löwel H, et al. KORA--a research platform for population based health research. Gesundheitswesen 2005;67 Suppl 1:19-25.

27 World Health Organization. Definition and diagnosis of diabetes mellitus and intermediate hyperglycaemia: report of a $\mathrm{WHO} /$ IDF consultation, 2006. Available: https://wwwwhoint/diabetes/ publications/diagnosis_diabetes2006/en/

28 Craney TA, Surles JG. Model-dependent variance inflation factor cutoff values. Qual Eng 2002;14:391-403.

29 Mathur A, Mohan V, Ameta D, et al. Aortic aneurysm. J Trans/ Int Med 2016;4:35-41.
30 Liu C-L, Ren J, Wang Y, et al. Adipocytes promote interleukin-18 binding to its receptors during abdominal aortic aneurysm formation in mice. Eur Heart J 2020;41:2456-68.

31 Sumida Y, Nakajima A, Itoh Y. Limitations of liver biopsy and noninvasive diagnostic tests for the diagnosis of nonalcoholic fatty liver disease/nonalcoholic steatohepatitis. World J Gastroenterol 2014;20:475.

32 Di Costanzo A, D'Erasmo L, Polimeni L, et al. Non-alcoholic fatty liver disease and subclinical atherosclerosis: a comparison of metabolically- versus genetically-driven excess fat hepatic storage. Atherosclerosis 2017;257:232-9.

33 McKimmie RL, Daniel KR, Carr JJ, et al. Hepatic steatosis and subclinical cardiovascular disease in a cohort enriched for type 2 diabetes: the diabetes heart study. Am J Gastroenterol 2008;103:3029-35.

34 Petit JM, Guiu B, Terriat B, et al. Nonalcoholic fatty liver is not associated with carotid intima-media thickness in type 2 diabetic patients. J Clin Endocrinol Metab 2009;94:4103-6.

35 Targher G, Bertolini L, Padovani R, et al. Non-alcoholic fatty liver disease is associated with carotid artery wall thickness in diet-controlled type 2 diabetic patients. J Endocrinol Invest 2006;29:55-60.

36 Stefan N, Häring $\mathrm{H}-\mathrm{U}$, Cusi K. Non-alcoholic fatty liver disease: causes, diagnosis, cardiometabolic consequences, and treatment strategies. Lancet Diabetes Endocrinol 2019;7:313-24.

37 Stefan N, Kantartzis K, Machann J, et al. Identification and characterization of metabolically benign obesity in humans. Arch Intern Med 2008;168:1609-16.

38 Roden M. Mechanisms of disease: hepatic steatosis in type 2 diabetes--pathogenesis and clinical relevance. Nat Clin Pract Endocrinol Metab 2006;2:335-48.

39 Bornfeldt KE, Tabas I. Insulin resistance, hyperglycemia, and atherosclerosis. Cell Metab 2011;14:575-85.

40 Eslam M, Newsome PN, Sarin SK, et al. A new definition for metabolic dysfunction-associated fatty liver disease: an international expert consensus statement. $J$ Hepatol 2020;73:202-9.

41 Ampuero J, Aller R, Gallego-Durán R, et al. The effects of metabolic status on non-alcoholic fatty liver disease-related outcomes, beyond the presence of obesity. Aliment Pharmacol Ther 2018;48:1260-70.

42 Qayyum A, Chen DM, Breiman RS, et al. Evaluation of diffuse liver steatosis by ultrasound, computed tomography, and magnetic resonance imaging: which modality is best? Clin Imaging 2009;33:110-5.

43 Brinjikji W, Huston J, Rabinstein AA, et al. Contemporary carotid imaging: from degree of stenosis to plaque vulnerability. $J$ Neurosurg 2016;124:27-42. 